\title{
SCHOTTKY'S FORM AND THE HYPERELLIPTIC LOCUS
}

\author{
CRIS POOR
}

(Communicated by Albert Baernstein II)

\begin{abstract}
We show that Schottky's modular form, $J_{g}$, has in every genus an irreducible divisor which contains the hyperelliptic locus. We also improve a corollary of Igusa concerning Siegel modular forms that must necessarily vanish on the hyperelliptic locus.
\end{abstract}

\section{$\S 1$. INTRODUCTION}

In 1888 Schottky gave the famous modular cusp form $J_{4}$ which vanishes on the Jacobian locus in $\mathcal{H}_{4}$, the Siegel upper half space of degree four. In 1981 Igusa represented $J_{4}$ as a rational multiple of $\vartheta_{D_{8}^{+}}^{2}-\vartheta_{D_{16}^{+}}$where $\vartheta_{D_{8}^{+}}$and $\vartheta_{D_{16}^{+}}$are the theta series associated to the lattices $D_{8}^{+}$and $D_{16}^{+}$, respectively. This representation could be "accidental" in that the dimension of cusp forms of weight 8 on $\mathcal{H}_{4}$ is small enough to make the the proportionality of forms arising from different sources likely. On the other hand this representation could point to a deeper relationship between differences of theta series and geometrically interesting loci in $\mathcal{H}_{g}$.

This paper provides a piece of data which supports the hypothesis of a deeper relationship. We show that $\vartheta_{D_{8}^{+}}^{2}-\vartheta_{D_{16}^{+}}$vanishes on the hyperelliptic locus in $\mathcal{H}_{g}$ for every degree $g$. If we view $\vartheta_{D_{8}^{+}}$and $\vartheta_{D_{16}^{+}}$as complete invariants of their associated lattices, then we see that Jacobians of hyperelliptic curves of any genus cannot distinguish the $D_{8}^{+} \oplus D_{8}^{+}$lattice from the $D_{16}^{+}$lattice. The proof we give is a simple modification of an argument due to Igusa in [3, page 845] that uses his homomorphism $\rho_{g}: A\left(\Gamma_{g}\right) \rightarrow S(2,2 g+2)$. Theorem 3.8 shows that if $f \in$ $A\left(\Gamma_{g}\right)$ vanishes at the cusps of the hyperelliptic locus, then $\rho_{g}(f)$ is divisible by the discriminant in $S(2,2 g+2)$. Theorem 3.8 provides a brief proof of the more interesting Corollary 3.10 that the modular form $\vartheta_{D_{8}^{+}}^{2}-\vartheta_{D_{16}^{+}}$always vanishes on the hyperelliptic locus. The author is presently investigating whether or not this form vanishes on the Jacobian locus for $g \geq 5$. I thank William Duke for the interesting talk at Columbia University on Siegel modular forms and codes which led me to this investigation. I also thank my colleague Armand Brumer for his explanations on these topics.

Received by the editors October 24, 1994 and, in revised form, January 30, 1995.

1991 Mathematics Subject Classification. Primary 11F46; Secondary 14K25, 11E45.

Key words and phrases. Analytic class invariant, theta series, hyperelliptic. 


\section{$\S 2$. Notation}

We first review the notation concerning modular forms and subvarieties of the moduli space of principally polarized abelian varieties. We let $\mathcal{H}_{g}$ denote the Siegel upper half space of degree $g \geq 1$, and let $\Gamma_{g}=\operatorname{Sp}_{g}(\mathbb{Z})$ denote the Siegel modular group which acts on $\mathcal{H}_{g}$. Let $A_{k}\left(\Gamma_{g}\right)$ denote the Siegel modular forms of weight $k$ for $\Gamma_{g}$, and let $A\left(\Gamma_{g}\right)=\bigoplus_{k \geq 0} A_{k}\left(\Gamma_{g}\right)$ be the graded ring of Siegel modular forms. For $g \geq 2$ a homomorphism of graded rings $\Phi_{g}: A\left(\Gamma_{g}\right) \rightarrow A\left(\Gamma_{g-1}\right)$ is defined for $f \in A\left(\Gamma_{g}\right)$ and $\Omega \in \mathcal{H}_{g-1}$ by

$$
\left(\Phi_{g} f\right)(\Omega)=\lim _{\lambda \rightarrow+\infty} f\left(\begin{array}{cc}
\Omega & 0 \\
0 & i \lambda
\end{array}\right) .
$$

Elements in the kernel of $\Phi_{g}$ are called cusp forms. We view $\mathcal{A}_{g}=\mathcal{H}_{g} / \Gamma_{g}$ as the moduli space of principally polarized abelian varieties. The Torelli map sends a compact Riemann surface of genus $g$ to its Jacobian's class in $\mathcal{A}_{g}$. We let $\mathcal{J}_{g}$ denote the closure in $\mathcal{A}_{g}$ of the image of the Torelli map and refer to $\mathcal{J}_{g}$ as the Jacobian locus. In the same way we let $h_{g}$ denote the closure of the image of the restriction of the Torelli map to hyperelliptic Riemann surfaces, and call $h_{g}$ the hyperelliptic locus. We say that a Siegel modular form $f \in A\left(\Gamma_{g}\right)$ vanishes on $h_{g}$ if for all $\Omega \in \mathcal{H}_{g}$ such that $[\Omega] \in h_{g}$ we have $f(\Omega)=0$.

We now discuss lattices in $\mathbb{R}^{n}$ and their associated theta series. A lattice $\Lambda \subseteq \mathbb{R}^{n}$ is called integral if for any $\ell_{1}, \ell_{2} \in \Lambda$ the value of the inner product $\left\langle\ell_{1}, \ell_{2}\right\rangle$ is an integer. The following sequence of analytic functions $\vartheta_{\Lambda}$ are invariant under isometries of the lattice $\Lambda$.

2.2 Definition. Let $\Lambda$ be a lattice in $\mathbb{R}^{n}$. For each $g \geq 1$ the theta series of $\Lambda$, $\vartheta_{\Lambda}: \mathcal{H}_{g} \rightarrow \mathbb{C}$, is defined for $\Omega \in \mathcal{H}_{g}$ by

$$
\vartheta_{\Lambda}(\Omega)=\sum_{\ell_{1}, \ldots, \ell_{g} \in \Lambda} \exp \left(i \pi \sum_{j, k=1}^{g} \Omega_{j k}\left\langle\ell_{j}, \ell_{k}\right\rangle\right)
$$

An integral lattice $\Lambda$ is called even if for all $\ell \in \Lambda$ we have $\langle\ell, \ell\rangle \in 2 \mathbb{Z}$. If $\Lambda$ is an even self-dual lattice of dimension $n$ we have $\vartheta_{\Lambda} \in A_{n / 2}\left(\Gamma_{g}\right)$ for each $g$. For such $\Lambda$ we necessarily have that 8 divides $n$, and the examples relevant here are the lattices $D_{n}^{+}$for $n \in 8 \mathbb{Z}^{+}$in the notation of Conway and Sloane [1, page 119]. For $n=8$ there is one isometry class of even self-dual lattices, given by $D_{8}^{+}$; for $n=16$ there are two isometry classes, given by $D_{8}^{+} \oplus D_{8}^{+}$and $D_{16}^{+}$. Theta series satisfy $\Phi_{g}\left(\vartheta_{\Lambda}\right.$ on $\left.\mathcal{H}_{g}\right)=\vartheta_{\Lambda}$ on $\mathcal{H}_{g-1}$.

2.3 Definition. For $g \geq 1$, define $J_{g} \in A_{8}\left(\Gamma_{g}\right)$ by $J_{g}=\vartheta_{\left(D_{8}^{+} \oplus D_{8}^{+}\right)}-\vartheta_{D_{16}^{+}}$.

From the work of Igusa in [4] we know that the vanishing of $J_{4}$ defines $\mathcal{J}_{4}$ in $\mathcal{A}_{4}$.

Finally, we shall use the standard notation $\mathbb{C}\left[a_{1}, \ldots, a_{r}\right], \mathbb{C}\left(a_{1}, \ldots, a_{r}\right)$ for polynomial domains and their quotient fields in $r$ variables. Let $\mathbb{C}\left[a_{1}, \ldots, a_{r}\right]^{\text {sym }}$ and $\mathbb{C}\left(a_{1}, \ldots, a_{r}\right)^{\text {sym }}$ denote, respectively, the polynomial domain and rational function field fixed under the action of the symmetric group $S_{r}$ on $\mathbb{C}(a$.) induced by permutations of $a_{1}, \ldots, a_{r}$. For $s \geq 0$, we denote by $\mathbb{C}[a .]_{s}^{\text {sym }}$ the polynomials in $\mathbb{C}[a .]^{\text {sym }}$ of degree $s$ in any one, hence in any, of the $a_{i}$. Elements of $\mathbb{C}[a .]_{s}^{\text {sym }}$ are said to have weight $s$. We note that $\mathbb{C}[a .]^{\mathrm{sym}}=\bigoplus_{s \geq 0} \mathbb{C}[a .]_{s}^{\mathrm{sym}}$ but that this is not the usual 
grading on $\mathbb{C}[a .]^{\mathrm{sym}}$ given by the homogeneous degree in the $a_{i}$; rather it is the grading given by the homogeneous degree in the elementary symmetric functions of the $a_{i}$. If we let $\Delta_{r}=\prod_{i<j}\left(a_{i}-a_{j}\right) \in \mathbb{C}\left[a_{1}, \ldots, a_{r}\right]$ as usual, then the element $\Delta_{r}^{2} \in \mathbb{C}\left[a_{1}, \ldots, a_{r}\right]_{s}^{\text {sym }}$ has weight $s=2(r-1)$.

\section{$\S 3 . \mathbf{J}_{g}$ VANISHes ON $\mathbf{h}_{g}$}

In this section we prove that a hyperelliptic cusp form of weight less than $8+4 / g$ must vanish on the hyperelliptic locus, $h_{g}$. The main tools are Igusa's homomorphism $\rho_{g}: A\left(\Gamma_{g}\right) \rightarrow S(2,2 g+2)$ from a subring of Siegel modular forms to a graded ring of "binary invariants", and Tsuyumine's map $T_{g}: S(2,2 g+2)-\rightarrow$ $\mathbb{C}\left(a_{1}, \ldots, a_{2 g}\right)$ that gives the $\rho$-induced action of $\Phi_{g}$ on $S(2,2 g+2)$.

3.1 Definition. For $r \geq 1, s \geq 0$, let $S(2, r)_{s}$ be the set of $f \in \mathbb{C}\left[a_{1}, \ldots, a_{r}\right]$ such that both 1 . and 2. hold.

1. For all $\left(\begin{array}{ll}a & b \\ c & d\end{array}\right) \in \mathrm{SL}_{2}(\mathbb{C})$ we have

$$
f\left(\frac{a a_{1}+b}{c a_{1}+d}, \ldots, \frac{a a_{i}+b}{c a_{i}+d}, \ldots, \frac{a a_{r}+b}{c a_{r}+d}\right)=\left(\prod_{i=1}^{r}\left(c a_{i}+d\right)\right)^{-s} f\left(a_{1}, \ldots, a_{i}, \ldots, a_{r}\right) .
$$

2. $f \in \mathbb{C}\left[a_{1}, \ldots, a_{r}\right]_{s}^{\mathrm{sym}}$.

3.2 Definition. For $r \geq 1$, let $S(2, r)=\bigoplus_{s \geq 0} S(2, r)_{s}$.

3.3 Remarks. If we apply condition 1. of Definition 3.1 to $\left(\begin{array}{ll}a & b \\ c & d\end{array}\right)=\left(\begin{array}{ll}1 & b \\ 0 & 1\end{array}\right)$ we see that $S(2, r) \subseteq \mathbb{C}\left[a_{i}-a_{j} \mid i, j \in\{1, \ldots, r\}\right] \cap \mathbb{C}\left[a_{1}, \ldots, a_{r}\right]^{\mathrm{sym}}$. Also notice that $\Delta_{r}$ satisfies condition 1 . with $s=r-1$.

3.4 Theorem (Igusa, [3, page 844]). Let $g \geq 1$. There exists a homomorphism of graded rings $\rho_{g}: \operatorname{Dom}\left(\rho_{g}\right) \subseteq A\left(\Gamma_{g}\right) \rightarrow S(2,2 g+2)$ such that conditions 1. -3 . hold.

1. $\operatorname{Dom}\left(\rho_{g}\right)$ contains all modular forms of even weight.

2. $\operatorname{Ker}\left(\rho_{g}\right)$ is the ideal of $\operatorname{Dom}\left(\rho_{g}\right)$ vanishing on $h_{g}$.

3. $\rho_{g}$ multiplies weights by $\frac{1}{2} g$.

3.5 Definition [7, page 762]. Let $g, m \geq 1$. Define $T_{g}: \bigoplus_{m \geq 1} S(2,2 g+2)_{g m} \rightarrow$ $\mathbb{C}\left(a_{1}, \ldots, a_{2 g}\right)$ by, for $I \in S(2,2 g+2)_{g m}$,

$$
\left(T_{g} I\right)\left(a_{1}, \ldots, a_{2 g}\right)=\left(\prod_{i=1}^{2 g} a_{i}\right)^{-m} I\left(a_{1}, \ldots, a_{2 g}, 0,0\right) .
$$

In [7] the domain of $T_{g}$ (designated by $\Phi$ in [7]) is given as $S(2,2 g+2)$ and the range space of $T_{g}$ is given as $\mathbb{C}\left(a_{1}, \ldots, a_{2 g}\right)$, but the formula defining $T_{g}$ gives $T_{g} I$ in the algebraic extension $\mathbb{C}\left(a_{1}, \ldots, a_{2 g}\right)\left(\sqrt[g]{a_{1} \ldots a_{2 g}}\right)$. One easy way to mend this discrepancy is to define $\operatorname{Dom}\left(T_{g}\right)=\bigoplus_{m \geq 1} S(2,2 g+2)_{g m}$ so that the range space of $T_{g}$ is $\mathbb{C}\left(a_{1}, \ldots, a_{2 g}\right)$. This is what has been done in Definition 3.5. The $\operatorname{Dom}\left(T_{g}\right)$ in Definition 3.5 includes the $\rho_{g}$-images of even weight modular forms; this inclusion is all we use here and all used in [7] to prove the following proposition. 
3.6 Proposition (Tsuyumine [7, page 786]). Let $g \geq 1$. There is a choice of $\rho_{g}$ in Theorem 3.4 such that for even $k$ and for all $f \in A_{k}\left(\Gamma_{g}\right)$ we have $\left(T_{g} \circ \rho_{g}\right)(f)=$ $\left(\rho_{g-1} \circ \Phi_{g}\right)(f)$ in $S(2,2 g)$.

3.7 Definition. Let $g \geq 2$, and let $f \in A\left(\Gamma_{g}\right)$. We say that $f$ is a hyperelliptic cusp form when $\Phi_{g}(f) \equiv 0$ on $h_{g-1}$.

3.8 Theorem. Let $k \in 2 \mathbb{Z}^{+}$. Let $f \in A_{k}\left(\Gamma_{g}\right)$ be a hyperelliptic cusp form. Then we have $\Delta_{2 g+2}^{2}$ divides $\rho_{g}(f)$ in $S(2,2 g+2)$.

Proof. Let $f$ be a hyperelliptic cusp form so that we have $\Phi_{g}(f) \equiv 0$ on $h_{g-1}$ by Definition 3.7. From 2. of Theorem 3.4 we have $\Phi_{g}(f) \in \operatorname{Ker}\left(\rho_{g-1}\right)$. From Tsuyumine's Proposition 3.6 we have $T_{g}\left(\rho_{g}(f)\right)=\rho_{g-1}\left(\Phi_{g}(f)\right)=0$ in $\mathbb{C}\left(a_{1}, \ldots, a_{2 g}\right)$. From the definition of $T_{g}$ we see that $\rho_{g}(f)$ is in the ideal $\left(a_{2 g+1}, a_{2 g+2}\right)$ of the ring $\mathbb{C}\left[a_{1}, \ldots, a_{2 g+2}\right]$. Since $\rho_{g}(f) \in S(2,2 g+2) \subseteq \mathbb{C}\left[a_{i}-a_{j} \mid i, j \in\{1, \ldots, 2 g+2\}\right]$, we have $\left(a_{2 g+1}-a_{2 g+2}\right)$ divides $\rho_{g}(f)$ in $\mathbb{C}[a$. $]$. Since $\mathbb{C}[a$. $]$ is a unique factorization domain, the facts that $\rho_{g}(f)$ is divisible by $\left(a_{2 g+1}-a_{2 g+2}\right)$ and that $\rho_{g}(f) \in \mathbb{C}[a \text {. }]^{\mathrm{sym}}$ imply that $\Delta_{2 g+2}$ divides $\rho_{g}(f)$ in $\mathbb{C}[a$.$] . Since \frac{\rho_{g}(f)}{\Delta_{2 g+2}}$ is an alternating polynomial in the $a$., we have that $\Delta_{2 g+2}$ divides $\frac{\rho_{g}(f)}{\Delta_{2 g+2}}$ in $\mathbb{C}[a$. $]$. Since $\Delta_{2 g+2}^{2}$ and $\rho_{g}(f)$ are both in $S(2,2 g+2)$, their quotient is as well, and we have that $\Delta_{2 g+2}^{2}$ divides $\rho_{g}(f)$ in $S(2,2 g+2)$.

3.9 Corollary. Let $k \in 2 \mathbb{Z}^{+}$. Let $f \in A_{k}\left(\Gamma_{g}\right)$ be a hyperelliptic cusp form. If $k<8+\frac{4}{g}$, then $f$ vanishes identically on $h_{g}$.

Proof. We have that $\Delta_{2 g+2}^{2}$ divides $\rho_{g}(f)$ in $S(2,2 g+2)$ by Theorem 3.8. The weight of $\Delta_{2 g+2}^{2}$ is $2(2 g+1)$ and the weight of $\rho_{g}(f)$ is $\frac{1}{2} g k$ when $\rho_{g}(f)$ is nontrivial. However, we have $\frac{1}{2} g k<2(2 g+1)$ by hypothesis so that $\rho_{g}(f)$ being divisible by $\Delta_{2 g+2}^{2}$ implies that $\rho_{g}(f)=0$. Therefore we have $f \equiv 0$ on $h_{g}$ by Igusa's Theorem 3.4 .

3.10 Corollary. For all $g \geq 1$, the Siegel modular form $J_{g}$ vanishes on the hyperelliptic locus $h_{g}$.

Proof. For $g$ such that $1 \leq g \leq 4$ this is known from the work of Witt [8], Kneser and Igusa [4], [5]. Corollary 3.10 follows by induction on $g$. Suppose that we have $J_{g} \equiv 0$ on $h_{g}$; then we have $\Phi_{g+1}\left(J_{g+1}\right)=J_{g} \equiv 0$ on $h_{g}$ so that $J_{g+1}$ is a hyperelliptic cusp form. $J_{g+1}$ is of even weight 8 so that we may apply Corollary 3.9 to conclude that $J_{g+1} \equiv 0$ on $h_{g+1}$.

3.11 Remark. Corollary 3.10 may also be proven using Thomæ's formula [3, pg. 838] and the theta identities in Lemma 1 of [5, pg. 354]. It then reduces to the following interesting polynomial identity which can be proven inductively by letting $a_{2 g+1}=a_{2 g+2}$. For $g \geq 1$ we have the polynomial identity in $\mathbb{Z}\left[a_{1}, \ldots, a_{2 g+2}\right]$ :

$$
\begin{aligned}
\left(\sum_{\left\{T \amalg T^{\mathrm{c}}\right\}} \prod_{i<j ; i, j \in T}\left(a_{i}-a_{j}\right)^{2} \prod_{i<j ; i, j \in T^{\mathrm{c}}}\left(a_{i}-a_{j}\right)^{2}\right)^{2} & \\
& =2^{g} \sum_{\left\{T \amalg T^{\mathrm{c}}\right\}} \prod_{i<j ; i, j \in T}\left(a_{i}-a_{j}\right)^{4} \prod_{i<j ; i, j \in T^{\mathrm{c}}}\left(a_{i}-a_{j}\right)^{4} .
\end{aligned}
$$


The above sum is over the $\frac{1}{2}\left(\begin{array}{c}2 g+2 \\ g+1\end{array}\right)$ partitions $T \amalg T^{\mathbf{c}}$ of $\{1,2, \ldots, 2 g+2\}$ for which both $T$ and $T^{\mathbf{c}}$ have $g+1$ elements. This formula was our original method of proof and was also noted by the referee.

Finally, we mention that $J_{g}$ is irreducible in $A\left(\Gamma_{g}\right)$.

3.12 Proposition. For all $g \geq 4$, the divisor of $J_{g}$ in $\mathcal{A}_{g}$ is irreducible.

Proof. We will show by induction on $g$ that the divisor of $J_{g}$ is proper and irreducible in $A\left(\Gamma_{g}\right)$ for $g \geq 4$. The case $g=4$ is due to Igusa [4]. We use a principle of Freitag which he deduces from his "Satz 2" in [2, page 256]. "For $g \geq 3$ an $f \in A\left(\Gamma_{g}\right)$ has an irreducible divisor, $\operatorname{div}(f)$, if $f$ may not be written as the product of modular forms of lower weight." If we had $J_{g}=\psi_{1} \psi_{2}$ in $A\left(\Gamma_{g}\right)$ where $0<\operatorname{deg} \psi_{1}, \operatorname{deg} \psi_{2}<8$, then applying the map $\Phi_{g}$ to $J_{g}$ and using the induction hypothesis show that $J_{g}$ is nontrivial on $\mathcal{H}_{g}$ and that $J_{g-1}=\Phi_{g}\left(\psi_{1}\right) \Phi_{g}\left(\psi_{2}\right)$ in $A\left(\Gamma_{g-1}\right)$ where $0<\operatorname{deg} \Phi\left(\psi_{1}\right), \operatorname{deg} \Phi\left(\psi_{2}\right)<8$. This is impossible because $\operatorname{div}\left(J_{g-1}\right)$ is irreducible by the induction hypothesis. This shows that $\operatorname{div}\left(J_{g}\right)$ is both proper and irreducible in $\mathcal{A}_{g}$.

\section{REFERENCES}

1. J.H. Conway, and N.J.A. Sloane, Sphere Packings, Lattices and Groups, Grund. der math. Wiss. 290, Springer-Verlag, New York, 1993. MR 93h:11069

2. E. Freitag, Die Irreduzibilität der Schottkyrelation (Bemerkung zu einen Satz von J. Igusa), Arch. Math. 40 (1983), 255-259. MR 84m:14034

3. J. I. Igusa, Modular forms and projective invariants, Amer. J. Math. 89 (1967), 817-855. MR 37:5217

4. J. I. Igusa, On the irreducibility of Schottky's divisor, J. Fac. Sci. Univ. Tokyo Sect. IA Math. 28 (1981), 531-545. MR 83f: 14023

5. J. I. Igusa, Schottky's invariant and quadratic forms, Christoffel Symposium, Birkhäuser Verlag, 1981, pp. 352-362. MR 83m:10031

6. J. I. Igusa, Theta Functions, Grundlehren der math. Wiss., Springer Verlag 194, 1972. MR 48:3972

7. S. Tsuyumine, On Siegel modular forms of degree three, Amer. J. Math. 108 (1986), 755-862, 1001-1003. MR 88a:11047a,b

8. E. Witt, Eine Identität zwischen Modulformen zweiten Grades, Abhandlungen aus dem Math. Sem. Hansischen Universität 14 (1941), 323-337. MR 3:163d

Department of Mathematics, Fordham University, Bronx, New York 10458

E-mail address: poor@murray.fordham.edu 